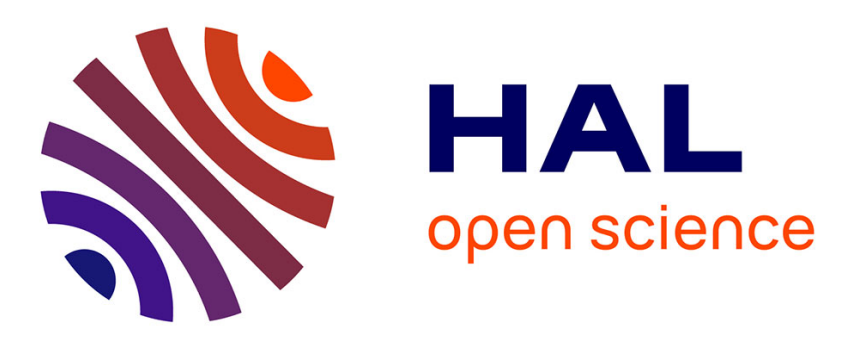

\title{
On Envelope-Tracking for SOA Amplification of Multicarrier Signals
}

J. C. Ortiz Cornejo, S Bejan, Stéphane Azou, J. A. Pardinas Mir, Pascal Morel

\section{To cite this version:}

J. C. Ortiz Cornejo, S Bejan, Stéphane Azou, J. A. Pardinas Mir, Pascal Morel. On Envelope-Tracking for SOA Amplification of Multicarrier Signals. 2017 IEEE International Symposium on Circuits and Systems (ISCAS), 2017, Baltimore, MD, USA, France. 10.1109/ISCAS.2017.8050451 . hal-01492351

\section{HAL Id: hal-01492351 https://hal.science/hal-01492351}

Submitted on 18 Mar 2017

HAL is a multi-disciplinary open access archive for the deposit and dissemination of scientific research documents, whether they are published or not. The documents may come from teaching and research institutions in France or abroad, or from public or private research centers.
L'archive ouverte pluridisciplinaire HAL, est destinée au dépôt et à la diffusion de documents scientifiques de niveau recherche, publiés ou non, émanant des établissements d'enseignement et de recherche français ou étrangers, des laboratoires publics ou privés. 


\title{
On Envelope-Tracking for SOA Amplification of Multicarrier Signals
}

\author{
J. C. Ortiz Cornejo*, S. Bejan ${ }^{\dagger}$ S. Azou ${ }^{\ddagger}$, J. A. Pardinas Mir *, P. Morel ${ }^{\ddagger}$ \\ * ITESO, Guadalajara, Mexico \\ ${ }^{\dagger}$ Military Technical Academy, Bucharest, Romania \\ ${ }^{\ddagger}$ ENIB / CNRS UMR 6285 Lab-STICC, Brest, France
}

\begin{abstract}
Semiconductor Optical Amplifiers are known to be prone to nonlinear effects in the saturated regime. This is particularly true if the signals to be amplified have a non-constant envelope and a high Peak-to-Average Power Ratio (PAPR). The benefits of envelope-tracking is investigated here for linearizing a Coherent-Optical OFDM (CO-OFDM) transmitter employing a SOA as a power booster. The effect of a filtered envelope is studied and its optimum amplification gain is evaluated by considering an accurate physical model of the SOA. Moreover, the joint combining of PAPR reduction (via nonlinear companding) and envelope tracking is proved to be effective, with a power margin close to $5 \mathrm{~dB}$.
\end{abstract}

Index Terms-Coherent Optical OFDM, Semiconductor optical amplifier (SOA), Linearization, Current Control, Envelope Tracking, PAPR reduction.

\section{INTRODUCTION}

Coherent Optical Orthogonal Frequency Division Multiplexing (CO-OFDM) has been the subject of an intense research effort in recent years [1][2]. This modulation technology offers several important advantages including high spectral efficiency, inherent robustness against intersymbol interference (ISI) caused by chromatic dispersion (CD) and polarization mode dispersion (PMD) and flexible digital signal processingbased implementation. However, a major drawback of multicarrier modulation schemes is their high sensitivity to system nonlinearities, as a result of large PAPR [5]. In this paper, we consider a CO-OFDM transmitter employing a Semiconductor Optical Amplifier (SOA), which has been recently pointed out as a good choice for boosting the power [3], due to features such as large optical bandwidth, small size and possibility of integration at limited cost [4]. However, as for most radio systems, an operating point close to the saturated region is associated to intermodulation distortions in presence of high PAPR, which can significantly degrade the bit-error-rate (BER) performance. Although PAPR reduction can partly alleviate the problem, it has no effect on the inherent nonlinear behavior of the SOA. So, the joint use of PAPR reduction and linearization should be considered for achieving the best transmitter performance. A wide variety of linearization techniques are discussed in the literature for reducing the nonlinear effects associated to optical communication systems [6]. An envelopetracking (ET) approach is investigated here for improving linearity in the SOA-based transmitter, with an eventual use of crest factor reduction via a simple $\mu$-law companding. The ET technique has been identified as a promising scheme for improving the power efficiency of broadband wireless systems [8] by modulating the supply voltage of the power amplifier according to the input envelope variations. The linearization capability of such technique for a SOA-based system has also been pointed out in [9], where the amplifier has its bias current controlled by the photo-detected input signal envelope. By using some theoretical developements, the authors showed that the current modulation translates into a constant carrier density within the active region and an increased effective saturation power of the amplifier. The practical feasibility of the scheme has been demonstrated by a two-tone experiment. The present paper aims at evaluating the benefits of the ET technique for advanced modulation formats like CO-OFDM. Our setup will be different to that considered by Saleh et al. in the sense that the SOA is used as a power booster and thus no photo-detector is required for implementing the ET scheme. Moreover, a more detailed evaluation of the filtering effects in the ET branch is proposed and the interest of combining a PAPR reduction with ET is revealed.

\section{System Model}

Our implementation relies on a self-developed SOA model [3], which has been fitted to simulate a commercially available bulk $750 \mu \mathrm{m}$ long SOA (INPHENIX-IPSAD1501), so that it yields a very good matching between simulated results and experimental results. The overall structure of the transmitter is illustrated in Fig. 1; it is a classical layout featuring an optional PAPR reduction block via nonlinear companding so as to adjust the statistics and the peak power of the $M$ QAM OFDM signal (with $N_{s c}$ subcarriers) to be amplified. For its simplicity, with only one parameter of the companding function, the $\mu$-law technique [7] will be adopted here. The Mach-Zehnder IQ modulator has a half-wave voltage $V_{\pi}$ of 6 $\mathrm{V}$ and a $1540 \mathrm{~nm}$ continuous-wave light applied at its input.

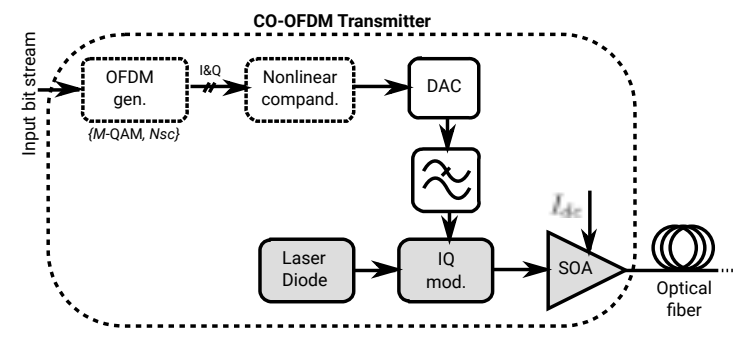

Fig. 1: CO-OFDM transmitter with SOA amplification 
The receiver, not illustrated, then performs a coherent detection, followed by various operations including time synchronization, nonlinear decompanding, equalization and digital demodulation. In the remaining of the study, a backto-back performance evaluation will be considered, with the assumption of a perfect coherent detection and by neglecting the digital-to-analog converter (DAC) effects. The case of a $5 \mathrm{GHz}$ electrical bandwidth 4-QAM CO-OFDM transmission spread over $N_{s c}=128$ subcarriers will be considered, with an oversamping factor of 4 .

\section{LINEARIZATION VIA ENVELOPE TRACKING}

The general idea behind envelope-tracking is to dynamically adjust the power supply signal so as to track the input envelope fluctuations. The dynamic supply involves an envelope amplifier (EA) with gain $G_{c}$ aiming at delivering the desired supply signal in a synchronous manner and in real-time which can be challenging in presence of multicarrier signals with large PAPR, occuring an envelope bandwidth far larger than that of the original baseband complex signal. Hence, shaping schemes are usually required for adapting the envelope dynamics to the application objectives (linearization, power efficiency, isogain,...) [8]. Our ET-based CO-OFDM transmitter setup is depicted in figure 2.

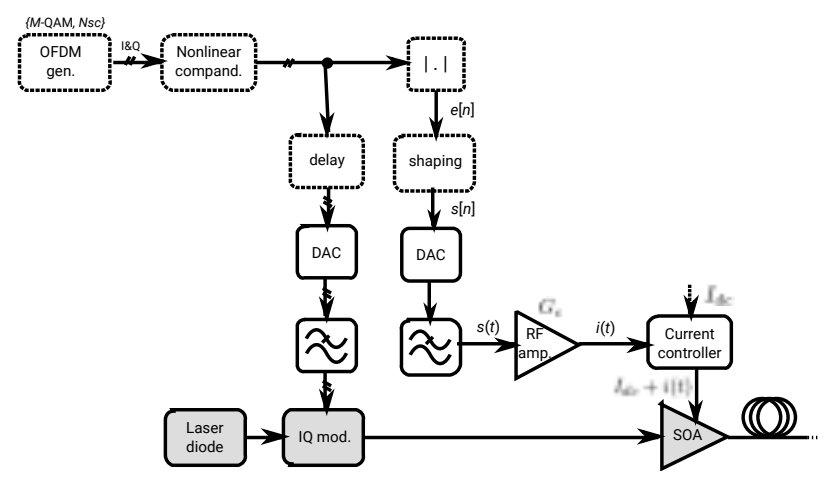

Fig. 2: CO-OFDM transmitter including an enveloppe-tracking branch for current control and a nonlinear companding block for PAPR reduction

As the SOA operates as a booster, the time-alignment, the envelope signal generation together with the envelope shaping can directly be processed in digital domain with no need of photo-detector, as would be the case for an inline amplifier. Theoretically, the amplifier can be perfectly linearized by adjusting the biasing current proportionally to the squared optical field envelope [9]. The resulting control signal may have frequency components over a few tens of $\mathrm{GHz}$, but due to the specific carrier density dynamics of the SOA (of the order of $0.3 \mathrm{~ns}$ ) a bandwidth limited to a few $\mathrm{GHz}$ may be sufficient for achieving the linearization. The practical feasibility of the concept has been shown by Saleh et al. for a two tone system only, with a $2 \mathrm{GHz}$ bandwidth baseband amplifier in the ET branch. In the present study, we investigate the effect of a 128-subcarriers OFDM envelope signal with a bandwidth reduced up to a lower limit of $625 \mathrm{MHz}$ (Fig. 3 , with the view to simplify as much as possible the implementation of the current control. Considering that the original
OFDM signal has a $5 \mathrm{GHz}$ bandwidth, this corresponds to a severe filtering effect. Thus, the shaped signal is expressed as $s[n]=h[n] * e[n]$, where $e[n]$ denotes the OFDM signal envelope and $h[n]$ is the low-pass filter impulse response (a 48th order FIR filter being chosen in the sequel); the resulting signal is then normalized in amplitude before its digital-toanalog conversion. The corresponding analog signal $s(t)$ is then adjusted in amplitude with a gain $G_{c}=\alpha I_{d c}$ depending on the amount of additional current required, where $\alpha \ll 1$ and $I_{d c}$ denoting the biasing current DC value. The resulting signal $i(t)=G_{c} s(t)$ will then be used to dynamically adjust the biasing current value. Throughout the sequel, a perfect synchronization will be assumed between the optical signal entering the SOA and the control signal $i(t)$.
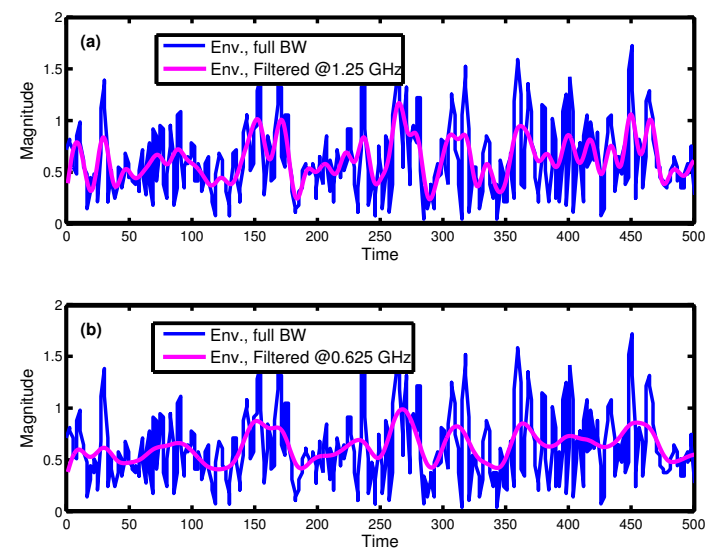

Fig. 3: Original envelope and reduced bandwidth envelope; (a) $1.25 \mathrm{GHz}$ bandwidth (b) $625 \mathrm{MHz}$ bandwidth

In addition to the ET scheme, aiming at linearizing the optical amplifier, a companding transform is eventually applied for reducing the PAPR of the signal to be amplified. According to this approach the original signal samples $x_{n}$ have their amplitude modified by a particular nonlinear function $f$, the resulting signal $y_{n}=f\left(x_{n}\right)$ is then converted into analog waveform. At the receiver side, the noisy signal $r_{n}=y_{n}+v_{n}$ is then transformed by the de-companding function in order to recover original signal (plus noise): $x_{n}=f^{-1}\left(r_{n}\right) \simeq$ $x_{n}+f^{-1}\left(v_{n}\right)$. For its simplicity, the $\mu$-law function expressed as follows is adopted:

$$
f\left(x_{n}\right)=A \operatorname{sgn}\left(x_{n}\right) \frac{\log \left(1+\mu \frac{x_{n}}{A}\right)}{\log (1+\mu)}
$$

where sign stands for the signum function and $\mu=1,2,3, \ldots$ denotes the key parameter for shaping the resulting probability distribution function (PDF). Increasing $\mu$ tends to boost the amount of medium amplitudes while compressing small and large amplitudes. The companding function together with the corresponfing PDF are depicted in Fig. 4, for the particular value $\mu=2$ which will be adopted in the sequel.

\section{NuMERICAL RESULTS}

The performance of the ET-based CO-OFDM transmitter is evaluated in this section for a $5 \mathrm{GHz}$ bandwidth 4-QAM transmission spread over $N_{s c}=128$ subcarriers (resulting in a 

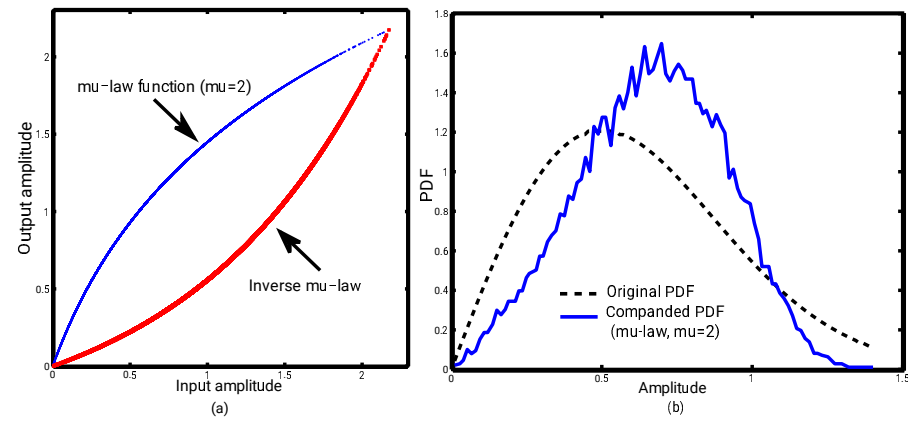

Fig. 4: (a) $\mu$-law function and its inverse for $\mu=2$; (b) Original PDF and companded signal distribution ( $2^{12}$ QAM symbols)

data rate of around $9 \mathrm{~Gb} / \mathrm{s}$ ). The scenario of an envelope lowpass-filtered at $1.25 \mathrm{GHz}$ in absence of companding has first been examined, over a wide range of SOA input power $P_{i n}$ and for a current gain $G_{c}$ going up to $0.5 I_{d c}$, with $I_{d c}=150 \mathrm{~mA}$. For evaluating the performance, the classical criterion of Error Vector Magnitude (EVM) [10] has been calculated for each couple $\left(P_{i n}, G_{c}\right)$ in a back-to-back configuration (receiver placed at SOA output). Due to the large computation time required for exploring the two-dimensional (2D) space, a short sequence made of $2^{9} 4$-QAM symbols has been used for this purpose (with a PAPR going up to $10.3 \mathrm{~dB}$ ). The results are given in figure 5 , where it can be clearly observed that low EVM values are achieved via current control over a wide range of $P_{i n}$.

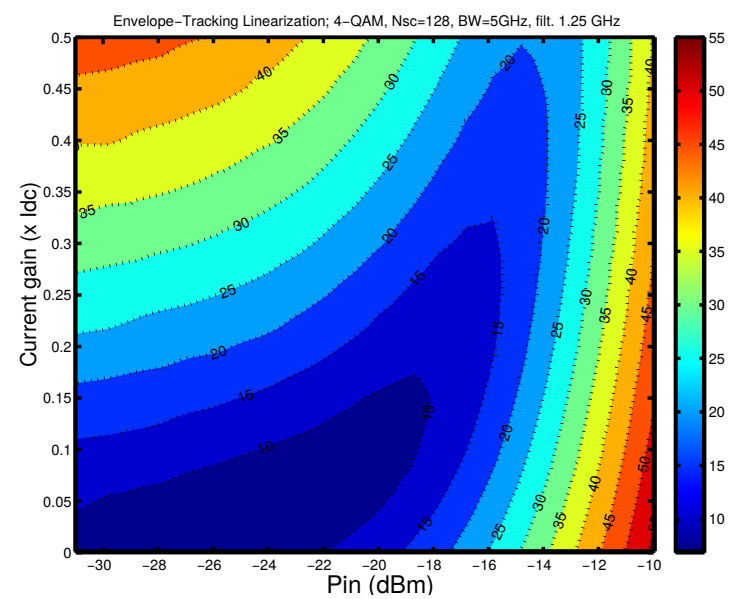

Fig. 5: EVM versus current gain $(\alpha)$ and SOA input power $\left(P_{i n}\right)$, for a bandwidth reduced at $1.25 \mathrm{GHz}$

For example, if an EVM limit of $30 \%$ is set (corresponding to a Bit-Error-Rate of $10^{-3}$ ), it can be seen that $P_{i n}$ can be increased up to $-12 \mathrm{dBm}$. It is also noticed that as more power is injected into the amplifier, a larger gain $G_{c}$ is required for compensating the nonlinear effects, particularly above -20 $\mathrm{dBm}$. An acceptable EVM value can eventually be ensured at high saturation $\left(P_{i n}>-12 \mathrm{dBm}\right)$, but with very large current gain.

In a similar way, EVM has been evaluated for each couple $\left(P_{i n}, G_{c}\right)$ when the envelope signal undergoes a bandwidth reduction at $1.25 \mathrm{GHz}$ and when a $\mu$-law companding is jointly used with the reduced bandwidth (BW) ET technique.

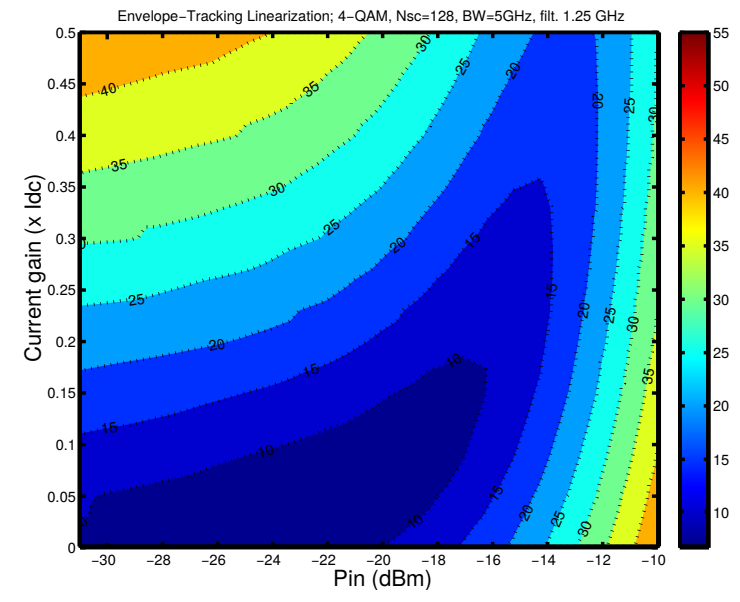

Fig. 6: EVM versus current gain $(\alpha)$ and SOA input power $\left(P_{i n}\right)$, for a bandwidth reduced at $1.25 \mathrm{GHz}$ with joint companding/ET

From Fig. 6, it can be observed that this scheme significantly enlarges the power range over which an acceptable EVM value can be satisfied, while alleviating the current gain $G_{c}$. The same approach has then been followed with a much narrower filter bandwidth $(625 \mathrm{MHz})$, with companding or not. For all the different schemes, we estimated a performance bound corresponding to the current gain $G_{c}$ yielding the minimum EVM for a given input power. The results are shown in Fig. 7, where we can first observe a quick rise in EVM if no companding nor linearization is adopted, as we move towards the saturated region; the considered EVM limit value being reached at $P_{\text {out }} \simeq 2 \mathrm{dBm}$. If the ET technique is used with a reduced $\mathrm{BW}$ of $1.25 \mathrm{GHz}$, an improvement of around 3 $\mathrm{dB}$ is achieved at $30 \%$ of EVM. For a better linearization,

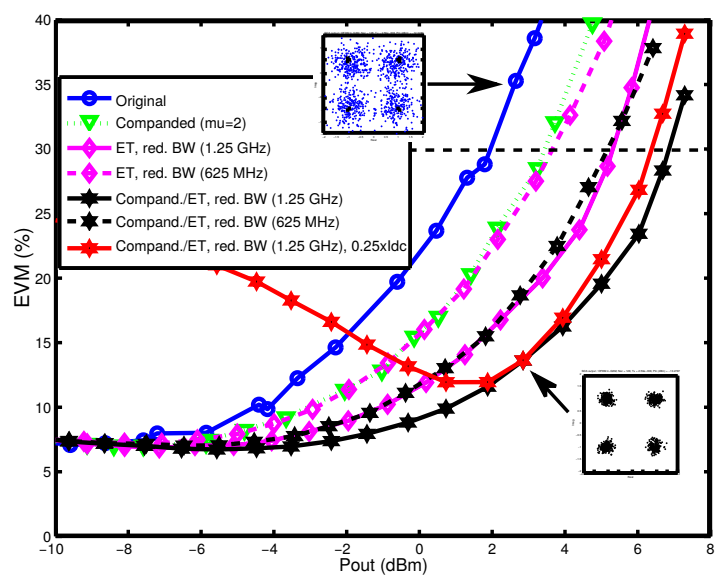

Fig. 7: EVM versus SOA output power

the joint use of PAPR reduction (with $\mu$-law companding here) and envelope tracking is an effective solution, as can be clearly seen on the same figure. An additional improvement close to $2 \mathrm{~dB}$ is achieved in this way in the SOA saturation region, with respect to the use of ET scheme alone. It can be also observed that this approach is far more effective than the use of $\mu$-law companding only, which just reduces the amount of nonlinear distortions by reshaping the statistics of the signal to be amplified. Note that a larger value of $\mu$ 
could significantly lower the EVM at high power, but at the price of less performance at low or medium power. Inserted constellations for an output power $P_{\text {out }}$ of $3 \mathrm{dBm}$ indicate a huge improvement of around $25 \%$ thanks to companding/ET compared to the original system. If a more severe filtering is applied (bandwidth of $625 \mathrm{MHz}$ ), a $1 \mathrm{~dB}$ penalty is measured for the ET scheme at $30 \%$ of EVM, with more degradation below this limit (the penalty going up to $2 \mathrm{~dB}$ ); the overall results being actually very close to those obtained with the $\mu$-law PAPR reduction alone. If the latter is combined with ET, it achieves a performance close to the ET scheme with a bandwidth reduced at $1.25 \mathrm{GHz}$. So, despite the relatively low bandwidth of the current control signal, an effective linearization is accomplished.

The optimum gain value against input power is plotted in figure 8 for the two ET schemes investigated (ET with reduced $\mathrm{BW}$ and $\mu$-law based ET with reduced $\mathrm{BW}$ ), with $\mathrm{BW}$ values of $1.25 \mathrm{GHz}$ and $625 \mathrm{MHz}$. Slowing down the envelope signal variation has an obvious effect on the current control, with lowered gain. It can be also observed that the usage of PAPR reduction significantly reduces the amount of additional current $i(t)$ required while achieving a better quality for the amplified signal if the optimum gain is chosen. The case of a current control with fixed gain $G_{c}=0.25 I_{d c}$ is illustrated in Fig. 7, when companding and reduced BW ET are employed (1.25 GHz case). In such conditions, the optimum bound is reached at $P_{\text {out }} \simeq 3 \mathrm{dBm}$ and the performance is still very good above this threshold, but a quick degradation is noticed as the operating point moves towards linear region (lower power and decreasing signal-to-noise ratio). This observation reveals that a self-calibrating ET technique should be designed so that the performance remains close to the optimum while the operating conditions (input power, modulation format, ...) change.

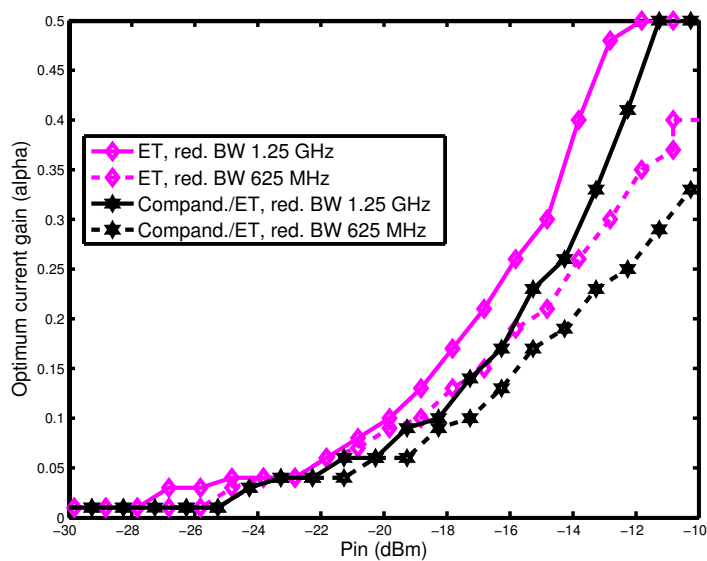

Fig. 8: Optimum gain ( $\alpha$ parameter) versus SOA input power

Finally, the dynamic AM/AM and AM/PM characteristics are depicted in Fig. 9 for the original system (subplots a, b) and for the companding/ET-based system (subplots c, d) with a reduced bandwidth at $1.25 \mathrm{GHz}$. The static nonlinearities together with memory effects can be clearly observed for the original system, whereas the proposed system exhibits a far better linearity. However, it is evident from plots $(\mathrm{c}, \mathrm{d})$ that the amplified signal is still affected by memory effects (spreaded points along straight lines).
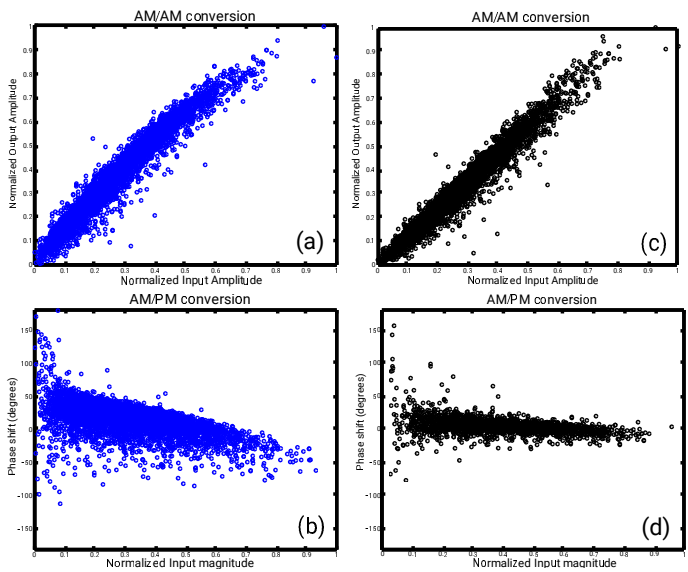

Fig. 9: $\mathrm{AM} / \mathrm{AM}$ and $\mathrm{AM} / \mathrm{PM}$ distortions corresponding to original signal (left) and after ET with reduced BW (right), respectively, for a SOA output power of $3 \mathrm{dBm}$

\section{CONCLUSION}

The benefits of envelope tracking have been investigated in this paper for mitigating the nonlinear effects in a coherent optical OFDM transmitter using a SOA as a power booster. To the knowledge of the authors, only Saleh et al. [9] have pointed out the effectiveness of the ET technique for linearizing a SOA, but by considering only a two-tone scenario. Here, the performance is evaluated for a complete CO-OFDM system, by employing an accurate physical model of the amplifier. Moreover, the impact of a filtered envelope is precisely evaluated; for a $5 \mathrm{GHz}$ OFDM signal, it is shown that the biasing current control is still effective at a bandwidth of $625 \mathrm{MHz}$, as a result of the simultaneous use of PAPR reduction via nonlinear companding with envelope tracking.

\section{REFERENCES}

[1] G. Zhang, M. D. Leenheer, A. Morea, and B. Mukherjee, "A survey on OFDM-based elastic core optical networking," IEEE Commun. Surveys Tuts., vol. 15, no. 1, pp. 6587, Feb. 2013.

[2] N. Cvijetic, "OFDM for Next-Generation Optical Access Networks," IEEE J. Lightw. Technol, vol. 30, no. 4, feb. 2012.

[3] H. Khaleghi, P. Morel, A. Sharaiha, and T. Rampone, "Experimental Validation of Numerical Simulations and Performance Analysis of a Coherent Optical-OFDM Transmission System Employing a Semiconductor Optical Amplifier,' IEEE J. Lightwave Technol., vol. 31, no. 1, pp. 161170, January 2013.

[4] M. J. Connelly, Semiconductor Optical Amplifiers. Boston, MA:Kluwer, 2002.

[5] Y. Rahmatallah and S. Mohan, "Peak-to-average power ratio reduction in OFDM systems: A survey and taxonomy," IEEE Communications Surveys and Tutorials, vol. 15, no. 4, 2013.

[6] X. Zhang, R. Zhu, D. Shen, and T Liu, "Linearization Technologies for Broadband Radio-Over-Fiber Transmission Systems," Photonics, vol. 1, no.4, pp. 455-472, 2014.

[7] H. Chen et al., "Nonlinear effect mitigation based on PAPR reduction using electronic pre-distortion technique in direct-detection optical OFDM system," Opt. Fiber Technol. 19 (October (5)) (2013) 387391.

[8] B. Kim, J. Kim, D. Kim, J. Son, Y. Cho, J. Kim, and B. Park, "Push the envelope: Design concepts for envelope-tracking power amplifiers," IEEE Microwave Mag., vol. 14, no. 3, pp. 6881, Mar. 2013.

[9] A. A.M. Saleh, R. M. Jopson, and T. E. Darcie, "Compensation of nonlinearity in semiconductor optical amplifiers," Electron. Lett., vol. 24 pp. 950-952, July 1988 .

[10] R. Schmogrow et al., "Error vector magnitude as a performance measure for advanced modulation formats", IEEE Photon. Technol. Lett., 24, 6163, 2012. 